\title{
Visibility Enhancement of Underwater Hazy Image using Multi-model SVD-DWT Fusion
}

\author{
Aditi Mehrolia \\ M. Tech. Scholar \\ Department of Computer Science and Engineering \\ LNCT, Bhopal
}

\begin{abstract}
Underwater hazy images (UHI) are inherently dark in nature and are affected by small suspending particles and marine snow. To increase the visibility range and vision depth, an artificial light is utilized. The rays of light are scattered by particles in the underwater medium and along with color attenuation results in problems such as contrast reduction, blurring of an image and color loss driving the images beyond recognition. In absence of any dehazing technique, the performance and usability of a standard enhancement algorithm may fail to produce desirable results. In this paper, we have proposed a novel solution to this problem by proposing fully automated underwater image dehazing using multimodal DWT fusion. Inputs for the combinational image fusion scheme are derived from Singular Value Decomposition (SVD) and Discrete Wavelet Transform (DWT) for contrast enhancement in HSV color space and color constancy using Shades of Gray algorithm respectively. The fused image is then subjected to contrast stretching operation to improve the global contrast and visibility of dark regions.
\end{abstract}

\section{Keywords}

Multi-model, DWT, SVD, Global Contrast, Visibility

\section{INTRODUCTION}

An enormous portion of our planet's surface is secured by seas and the soundness of our planet is administered by these water assets. The investigation of submerged vegetation is only a fundamental piece of maritime exploration work [1]. Submerged reviews highlight in logical applications, for example, paleohistory [2], topography [3], submerged ecological appraisal [4], and laying of significant distance gas pipelines and correspondence joins over the mainlands, request geo-referential studying [5] of the maritime bed. Maritime investigation is likewise worried about prospection of old wreck [6].

Dispersing is a significant issue which should be tended to while recuperating the dehazed picture from submerged pictures. As per the Jaffe-McGlamery submerged imaging model, the three segments, direct segment, forward dispersing segment and back-dissipating segment establish the absolute brilliance of a picture that movements towards the camera. The presence of natural and inorganic particles suspended in the volume of water, converged by the field of perspective on the camera and the enlightenment source is the reason for the dispersing marvel. This dispersing wonder is seriously influenced if turbidity is high. The level of dispersing relies upon shape and size attributes of particles. There are two sorts of dispersing, forward dissipating and in reverse dispersing as appeared in Figure 1.

\author{
Aditya Patel \\ Assistant Professor \\ Department of Computer Science and Engineering \\ LNCT, Bhopal
}

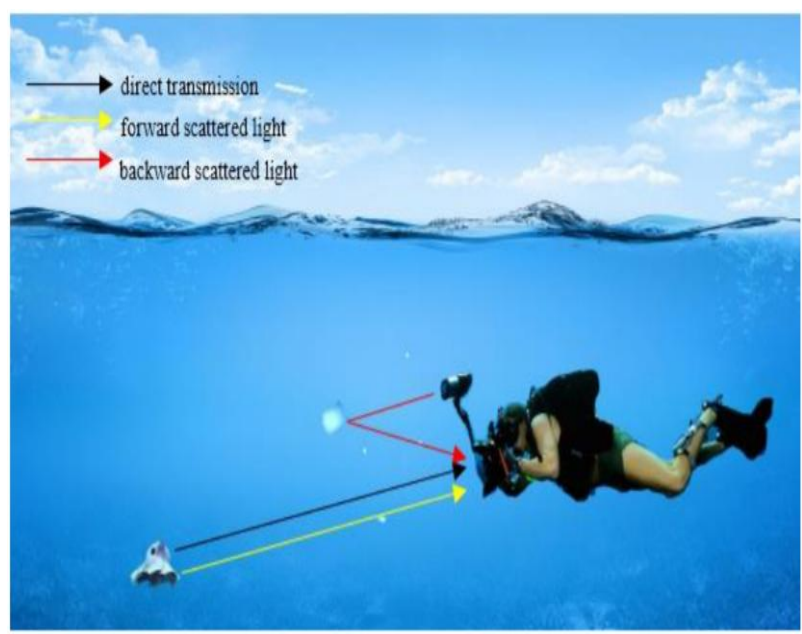

Fig. 1: Illustration of forward scatter, backward scatter and direct component

The forward scattering appears due to inter-reflections of local light among the particles in the path of the camera and underwater scene to be captured [7]. This contributes more significantly to the blurring of image and loss in contrast with color fidelity. Whereas the backward scattering is an additive noise in the form of marine snow patterns which appear owing to the reflection of the light particles towards the camera before reaching the object.

\section{IMAGE FUSION}

Picture handling is one sort of sign preparing for this picture goes about as information, it might be either photograph or video outline and the result of picture preparing might be either a picture or a bunch of attributes identified with the picture. The greater part of the picture preparing procedures, picture of two-dimensional sign is treated as information and standard sign handling methods are applied to it. Picture and video pressure is a functioning application region in picture handling. In the field of Image handling, picture combination has gotten a huge consideration for far off detecting, clinical imaging, machine vision and the military applications. A various leveled thought of picture combination has been proposed for joining critical data from a few pictures into one picture. The point of picture combination is to accomplish improved circumstance appraisal as well as more quick and exact finishing of a pre-characterized task than would be conceivable utilizing any of the sensors separately. Fundamentally picture combination requires exact methods and furthermore great comprehension of information. An answer for this issue is given by the twofold thickness complex DWT, which joins the qualities of the twofold thickness DWT and the double tree DWT. The twofold thickness complex DWT depends on two scaling capacities 
and four unmistakable wavelets, every one of which is explicitly planned with the end goal that the two wavelets of the primary pair are counterbalanced from one other by one half, and the other pair of wavelets structure a rough Hilbert change pair. By guaranteeing these two properties, the twofold thickness complex DWT has improved directional selectivity and can be utilized to actualize mind boggling and directional wavelet changes in various measurements. The prerequisite for the fruitful picture combination is that pictures must be effectively adjusted on a pixel-by-pixel premise. In this venture, the pictures to be joined are thought to be now completely enlisted. The Figure 1 shows the high level square chart of picture combination utilizing wavelet change. The two info pictures picture 1 and picture 2 that are caught from obvious and infrared camera individually are taken as data sources.

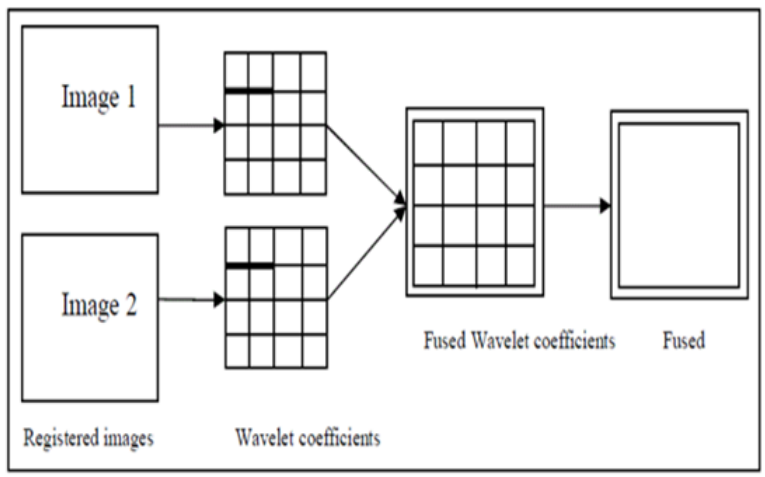

Fig. 2: Block diagram of DWT based image fusion

\section{PROPOSED METHODOLOGY}

Structure of proposed algorithm is representing by 3 . The I/P UHI is going through contrast enhancement (CE) and white balanced image (WBI). CE is scheme to gives image feature with different color. CE is also changing the value of color and find image feature. WBI is scheme to gives quality of object is white and removing nonsensical color casts to prevent reality which spring white in person. CE and WBI output get over 2-D DWT. 2-D DWT is CE and WBI O/P is fours sub-band and select LL sub-band. LL is more information and gets robustness.

The SVD contains brightening data in the picture with the goal that the change of the particular qualities will straightforwardly change enlightenment of the picture and other data present in the picture will stays same as in the past. The enlightment data is encircled in the LL sub band also, edges are packed in other sub groups. So isolating the highrecurrence sub groups and applying the brightening improvement in LL sub band will secure the edge data from conceivable debasement and afterward use of IDWT for recreation of picture will gives more keen picture.

The component of differentiation improvement can be credited to thresholding of DWT part and scaling of solitary estimations of LL coefficients. Since solitary [8] values indicate luminance of each picture layer after disintegration, scaling of these qualities prompts variety of luminance of each layer and subsequently prompts generally speaking differentiation upgrade.
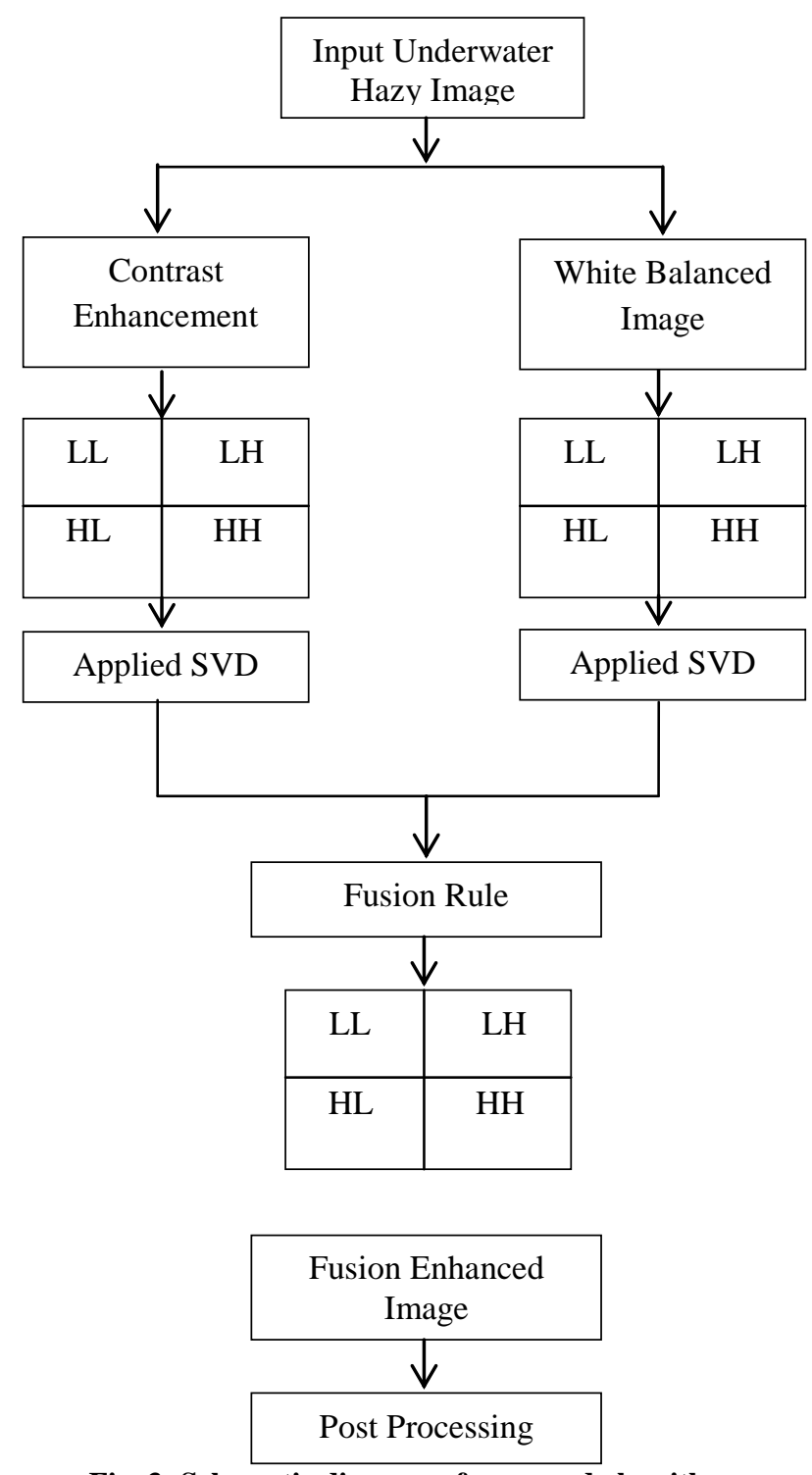

Fig. 3: Schematic diagram of proposed algorithm

\section{SIMULATION RESULT}

In this part, execution of proposed strategy utilizing SVD and DWT is assessed by thinking about constancy evaluation boundaries like mean, standard deviation, PSNR, MSE. Mean is the normal of all force esteem and higher mean indicates the great nature of picture. Standard deviation gives the normal differentiation of the picture. Better quality deviation demonstrates great differentiation of combined picture. The MSE speak to the aggregate squared blunder between the unique picture and remade picture.

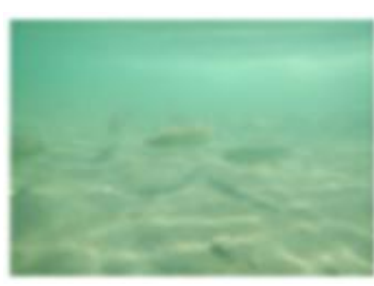

(a)

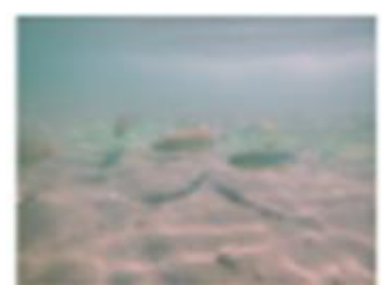

(b) 


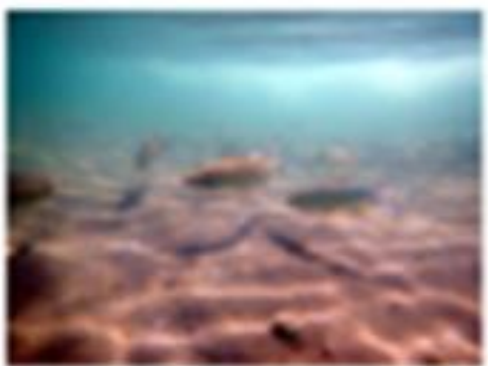

(c)

Fig. 4: UHI_1 (a) I/P Image (b) Enhancement Image (c) Output Image

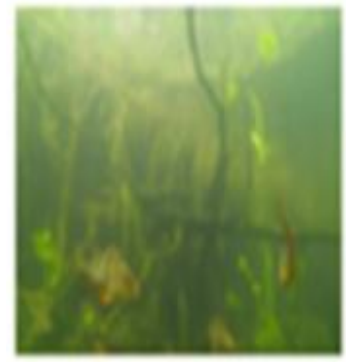

(a)

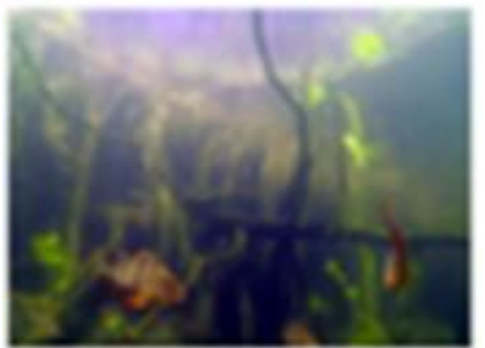

(c)

Fig. 5: UHI_2 (a) I/P Image (b) Enhancement Image (c) Output Image

The lower the estimation of MSE, the blunder might be lower. PSNR utilized for quality estimation proportion between unique picture and remade picture. The higher is PSNR, the better the nature of the recreated picture.

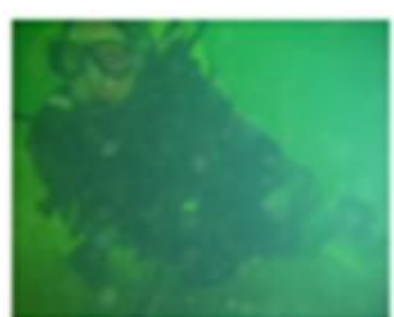

(a)

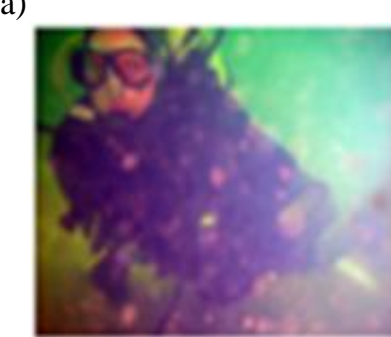

(c)

Fig. 6: UHI_3 (a) I/P Image (b) Enhancement Image (c) Output Image
Table 1: PSNR Value

\begin{tabular}{|c|c|}
\hline UHI_1 & $23.46 \mathrm{~dB}$ \\
\hline UHI_2 & $22.67 \mathrm{~dB}$ \\
\hline UHI_3 & $23.72 \mathrm{~dB}$ \\
\hline
\end{tabular}

Table 1: MSE Value

\begin{tabular}{|c|c|}
\hline UHI_1 & 3.564 \\
\hline UHI_2 & 4.719 \\
\hline UHI_3 & 3.902 \\
\hline
\end{tabular}

\section{CONCLUSION}

In this proposed work we have actualized basic yet powerful submerged single picture improvement method to address the issue of perceivability rebuilding and undesirable shading cast. The proposed strategy is carefully founded on non-actual model and as such doesn't request any already boundaries. Utilizing this methodology we prevailing with regards to planning calculation liberated from any priors, which are conventionally used to discover the profundity of murkiness. This proposed technique doesn't need any sort of computationally thorough channels which are generally used to refine the transmission maps in actual based picture development models.

Additionally, utilizing this proposed strategy we can defeat the impediments experienced already in a fix based submerged picture dehazing issues, for example, calculation of barometrical light worth, trouble with huge items like the shade of cloudiness and rules for determination of edge saving smoothing administrators.

\section{REFERENCES}

[1] Rajni Sethi, "Fusion of Underwater Image Enhancement and Restoration", International Journal of Pattern Recognition and Artificial Intelligence Vol. 34, No. 3 (2020) 2054007.

[2] H. Wang, R. Zhao, Y. Cen, L. Liang, Q. He, F. Zhang and $\mathrm{M}$. Zeng, Low-rank matrix recovery via smooth rank function and its application in image restoration, Int. J. Mach. Learn. Cybernet. 9 (2018) 1565-1576.

[3] Y. Wang, J. Zhang, Y. Cao and Z. Wang, A deep cnn method for underwater image enhancement, in 2017 IEEE Int. Conf. Image Processing (ICIP) (Beijing, China, 2017), pp. 1382-1386.

[4] C. O. Ancuti, C. Ancuti, C. D. Vleeschouwer and P. Bekaert, Color balance and fusion for underwater image enhancement, IEEE Trans. Image Process. 27 (2018) 379-393.

[5] C. Ancuti, C. O. Ancuti, T. Haber and P. Bekaert, Enhancing underwater images and videos by fusion, in 2012 IEEE Conf. Computer Vision and Pattern Recognition (CVPR) (2012), pp. 81-88.

[6] T. Çelebi and S. Ertürk, Visual enhancement of underwater images using empirical mode decomposition, Expert Syst. Appl. 39(1) (2012) 800-805. 
[7] L. Chao and M. Wang, Removal of water scattering, 2010 Int. Conf. Computer Engineering and Technology, Proceedings ICCET 2010, Vol. 2 (Chengdu, China, 2010), pp. 35-39.

[8] J. Y. Chiang and Y. C. Chen, Underwater image enhancement by wavelength compensation and dehazing, IEEE Trans. Image Process. 21(4) (2012) 1756-1769.

[9] A. Duarte, F. Codevilla, J. D. O. Gaya and S. S. C. Botelho, A dataset to evaluate underwater image restoration methods, in OCEANS 2016, Shanghai, April 2016, pp. 1-6.

[10] A. Galdran, D. Pardo, A. Picón and A. Alvarez-Gila, Automatic red-channel underwater image restoration, J. Vis. Commun. Image Represent. 26 (2015) 132-145.
[11] C. Gao, J. Zhou, C. Liu and Q. Pu, Image enhancement based on fractional directional derivative, Int. J. Mach. Learn. Cybern. 6(1) (2015) 35-41.

[12] X. Wei, H. Wang, G. Guo and H. Wan, Multiplex image representation for enhanced recognition, Int. J. Mach. Learn. Cybernet. 9 (2018) 383-392.

[13] J. Xiao, J. Hays, K. A. Ehinger, A. Oliva and A. Torralba, Sun database: Large-scale scene recognition from abbey to zoo, in 2010 IEEE Computer Society Conf. Computer Vision and Pattern Recognition (San Francisco, CA, USA, June 2010), pp. 3485-3492.

[14] C. Yan, N. Sang and T. Zhang, Local entropy-based transition region extraction and thresholding, Pattern Recognit. Lett. 24(16) (2003) 2935-2941. 